TM -456

0428

Evaluation of Matched Superconducting Dipoles

B. P. Strauss

D. F. Sutter

December 11, 1973

\begin{abstract}
Two superconducting Dual Dipole magnets have been measured at ac and dc excitations up to fields of $540 \mathrm{GeV}$ equivalent proton energy in the present NAL Main Ring. Apart from a quadrupole term of as yet undetermined origin, the measured quality of field on the midplane, $\Delta B / B o$, is consistent with the homogeniety requirements specified for these magnets. Additional field measurements and operational evaluation of magnet performance in a high radiation environment are being undertaken.
\end{abstract}


Introduction

Progress towards design of an Energy Doubler accelerator for the NAL Main Ring has been made by fabrication and testing of a series of small models culminating in magnetic testing of a matched pair of 29 inch long shell geometry dipole magnets. Known as the "Dual Dipoles", they were built to determine whether two superconducting magnets of a design being considered for the Energy Doubler could be made identical enough in vital characteristics to enable construction of a working accelerator. To this end the critical tests of pulsing both magnets simultaneously to determine their ac tracking properties and of measuring harmonic content of the dipole fields have been completed. Construction

The magnets are wound in concentric shells with the superconductor placed to best approximate a $\cos \theta$ current distribution. Design criteria for the wire placement required minimizing the sum of all harmonics up through the 22nd pole. Magnet characteristics are summarized in Table I. Coils are bonded to the bore tube and held against magnetic pressure forces by banding with 150 turns of 0.375 inches wide by 0.010 inch thick mylar tape. Longitudinal spacing between bands is 0.250 inches. By also using part of the mylar banding as a spacer between the inner shell and outer two shells, percolation cooling channels are provided throughout the windings so at least 40 percent of one narrow face of each turn is exposed to liquid helium. These are the first magnets at NAL that use graded conductor in the windings; that is, the distribution of superconducting wire throughout the coil is adjusted for critical current of the wire so that wire of lower characteristic $\mathrm{I}_{\mathrm{c}}$ is used in lower field regions. This has the promise of saving up to $25 \%$ in conductor costs over that of magnets using single $\mathrm{I}_{c}$ wire throughout. Projected design for the final 20 foot Doubler magnets required very high current density wire, $55 \mathrm{kA} / \mathrm{cm}^{2}$ and $75 \mathrm{kA} / \mathrm{cm}^{2}$; however, in order to expedite the magnet development program older, lower current density wire was used. Consequently, the maximum field in the bore of the Dual Dipoles is limited to 2. $7 \mathrm{~T}$ and a full field test of the suspension and banding systems could not be accomplished in this program. The higher current density wire is now on hand, and twenty 
foot Doubler prototype magnets are being built from it.

The magnet is held rigidly in its helium container by epoxy fiberglass G-10 spacers. This container forms the inner wall of the vacuum dewar and is held in place by a bicycle wheel type suspension system. The suspension "spokes" were wound with epoxy impregnated DuPont PRD-49 fiber. The suspension must repond with a net restoring force to displacement forces generated between the windings and iron shield when their respective axes are not coincident. Radiative thermal losses were reduced by winding 75 layers of super-insulation (aluminized mylar) around the helium jacket between the bicycle suspensions. Super-insulation was also interwoven between the spokes of the suspension. In the doubler the inner wall of the helium container, to which the windings are banded, will also form the wall of the evacuated beam tube. Because lab testing is facilitated by having ready access to the magnet bore, a $2^{\prime \prime}$ ID warm bore tube wrapped with 10 layers of super-insulation was installed before evacuating the cryostat. While this provides a slightly higher heat load it did not affect operation of the magnet.

Warm Testing - Quality Control

At the end of each stage of production the magnet coils were tested for shorts by ringing. Some layer to layer shorts were discovered as shells were being assembled onto the bore tube, but these were easily corrected and no further shorts observed. To verify the quality of insulation, hi-potting between coil and ground was done to a peak of 1500 volts at room temperature with no noticeable leakage current. Before sealing it in the dewar, the coil was fully instrumented with voltage taps and temperature sensing carbon resistors. Measurements were also made at room and liquid nitrogen temperatures of resistance and inductance. These results confirmed the values reported in Table $\mathrm{I}$.

Superconducting Operation

A general block diagram of the Dual Dipole operating system is shown in Figure 1. The power supply, an NAL standard Transrex 500-5, produces significant ripple and SCR noise necessitating the $\mathrm{L}$ section filter made with the Main Ring $\mathrm{B}_{2}$ magnet and 80 millifarad capacitor bank. Operation of the 
Dual Dipoles, which can be powered separately or in pairs, is the same as for conventional magnets up to a point. Turn on, turn off and other changes of current must be limited in rate or the magnet will quench, and when quenches do occur provision must be made for safely dispersing the energy stored in the magnetic field. It should be pointed out that the extraordinarily high quality thermal insulation necessary for normal operation means that most internally generated heat cannot be dissipated once the liquid helium is blown off. To dump the energy safely a sensitive voltage comparator differentially monitors the superconducting magnet and shuts off the Transrex when a resistive voltage develops across the magnet, indicating a quench. The collapsing field then introduces an emf of opposite polarity to the Transrex, forward biasing the shunt diode which is outside the dewar, and dumping the energy into a stainless steel load resistor.

During normal operation liquid Helium level is continuously monitored and automatically controlled via an in-line solenoid valve described previously. Ramping of the magnets is accomplished by programming the Transrex with a voltage signal resembling a standard Main Ring current waveform and derived from a commercial waveform generator and special shaping circuits.

Cooldown is initiated by passing liquid nitrogen directly into the dewar. By using the temperature dependence of the resistivity of the coil, the magnet is made to act as its own temperature sensor. At about $100^{\circ} \mathrm{K}$, before reaching thermal equilibrium with liquid Nitrogen, the nitrogen is thoroughly purged with helium gas. After a slight warming to ensure removal of all nitrogen, cooldown is continued with liquid Helium. From a warm start the entire drill requires less than three hours.

Magnet Performance

DC performance characteristics are reported in Table II. The maximum currents shown were reached only after much training, the process of attaining successively higher quench currents by repeated excitation of the magnet to the maximum current it will take. For the Dual Dipole four or five quench cycles at the start of each run were required to reach a constant maximum current. The training effects tended to be remembered in spite of 
recycling of the magnets to room temperature and so at the end of the test series both magnets were operating considerably closer to the short sample limits of the superconducting wire than in the beginning.

The ultimate limit for operation is set by short sample tests of the superconducting wire for critical current versus critical field at about $4.2^{\circ} \mathrm{K}$, the approximate temperature of the pool boiling Helium used to cool the Dual Dipoles. Practical operating limits are set by problems such as the $20 \%$ higher magnetic fields seen by the end coils that reduces critical current and the necessity, in the small geometry of the Doubler style magnet, of making extra short splices of the superconducting wire. With this latter problem in mind, special care was taken during manufacture to hold down, shunt with copper, heat sink and instrument all splices. During operation no voltage was observed across the splices to within the lower limits of our apparatus, $1 \mu \mathrm{V}$. The magnet could be ramped continuously at a 15 second cyclic period to $85 \%$ of its de critical current and reached a maximum dc current equal to $90 \%$ that of the smaller conductor as measured in a two dimensional cross section of the coil. This exceeds the design limits of $80 \%$ of short sample set for the Energy Doubler.

The transfer constant for each magnet was measured with a Varian NMR probe system and Hewlett Packard precision counter. Constants of the two magnets agree to within . $16 \%$ of each other and to within . $20 \%$ of the theoretically predicted values as reported in Table II. These values are an upper bound limited by the accuracy of the measurement. Magnetic Measurements

The small bore of these magnets and relatively large size of most precision magnetic measurement probes prevents adequate direct field mapping. Consequently, one must measure the harmonic content of the midplane with appropriate search coils and reconstruct the field shape from this data according to the relationship

$$
\mathrm{B}(r, \theta)=\mathrm{B}_{\mathrm{o}}\left(1+\sum_{\mathrm{n}=2}^{\infty} \mathrm{a}_{\mathrm{n}} \mathrm{r}^{\mathrm{n}-1} \cos \left(\mathrm{n} \theta+\alpha_{\mathrm{n}}\right)\right.
$$

where the $a_{n}$ are measured ratios of the higher order multipoles to the fundamental dipole. Two methods were used. In the first a coil is rotated at constant 
angular velocity and the output waveform fed into an electronic wave analyzer. The second method employs wire configurations uniquely sensitive to each harmonic of interest. As a rough check of these two techniques a transverse sweep with a Hall detector was made across the horizontal midplane of the bore of one magnet. The accuracy of data obtained with the Hall probe was only sufficient to allow a fit to a quadrupole field component which agrees reasonably well with the quadrupole term determined by the other two techniques.

\section{Rotating Coil}

The use of a rotating coil to measure magnetic fields is well known, 2 such devices have been built at $\mathrm{SLAC}^{3,4,5}$ and at $\mathrm{NAL}^{6}$ to determine the harmonic content of magnets. The NAL device was debugged as part of the Dual Dipole testing and several problems discovered and corrected. In particular, a large systematic error contributed by the mechanics of the first rotator assembly introduced abnormally large quadrupole and sextupole signals unrelated to the magnet under measurement. A second rotator solved this problem.

Coil output was coupled to a Quantech Model 2449 wave analyzer which measured the RMS value of the voltage at the harmonic frequencies corresponding to the higher order magnetic multipoles. No attempt was made to determine the phase angle of the higher order terms since the wave analyzer introduced a frequency dependent phase shift on the output signal which is reconstructed from the detected input harmonic. If this problem can be corrected or compensated, phase angles should be readily obtainable from this apparatus.

The system was checked for calibration as follows. A square wave of known frequency and amplitude was fed into the Quantech and the relative harmonic values were compared to the mathemactical Fourier analysis for the square wave. Significant departures from predicted values did not appear until after the 11 th harmonic ( 22 nd pole) which is well above that of present interest. The whole system was tested for instrumental error by rotating the coil in a Varian laboratory standard magnet. This magnet was previously mapped with an NMR probe and showed a variation in magnetic field of less than $50 \mathrm{mG}$ over a 2 " region at a field of $7 \mathrm{kG}$. The results of this test are presented in Table III. 
Since the pickup coil is only one inch long, measurements were made all along the axis of the magnet by mounting the rotating coil device on a lathe bed. Results obtained with this device are presented in Table IV. The average value for the central uniform region of the coil is presented. The midplane variation of field $\triangle B / B_{O}$ vs. the radial distance from the center is plotted in Figures 2 and 3 for all the multipoles measured except the quadrupole. The phase angles for each term are assumed to be those of the Snowden prediction which is also plotted in these figures. Addition of the quadrupole term will skew the field plot with respect to the $\mathrm{x}$ axis. "Stationary" Harmonic Analysis

Harmonic measurements were also performed using a coil wound with turns placed in simple $\cos n \theta$ approximations to the multipole of interest. The coil design is based on the work of Morgan ${ }^{7}$ and the Sampson group at Brookhaven. Spacing restrictions on our 1" diameter coil, required by the extra small warm bore of the doubler magnets, limited the coil to two dipole, a quadrupole, sextupole and decapole windings. ${ }^{8}$

Measurement of a harmonic consists of rotating the coil through $360^{\circ}$ in $8^{\circ}$ steps. The output of the harmonic coil of interest is then run through a precision integrator and converted to a digital value in a Fluke 8200 DVM, which can resolve $1 \mu \mathrm{V}$. At the end of each $8^{\circ}$ incremental rotation the DVM value and angle are recorded. The tabulated function so generated represents the incremental flux intercepted by the coil from 0 to $\theta$. A perfect $\cos n \theta$ winding will see no lower order harmonics, no higher order harmonics of even multipoles and small amplitudes of odd multipoles of the design harmonic. In an attempt to get larger voltages from the small, $1.25 \mathrm{~cm}$, radius coil, the windings were made with a cable-laid 7 strand wire which was interconnected to give 7 turns for each harmonic coil. Unfortunately, this is a difficult mechanical assembly and resulted in considerable dipole pickup on all coils. The data could still be used by Fourier analyzing the measurements for each harmonic coil and extracting appropriate amplitudes and phase angles. The amplitudes extracted are then adjusted for radius and compared to those obtained from the rotating coil. The data and comparisons are shown in Table IV and a reconstruction of the deviation from a dipole field, again excluding the quad- 
rupole data, is again shown in Figures 2 and 3 with the phase angle assumption used before.

As can be seen in Figures 2 and 3, the measurement shows that the worst deviation from a pure dipole on the midplane is \pm 6 parts in $10^{4}$ - again excluding the quadrupole component. Both measurements confirm this. The computer predictions tended towards a better chromaticity closer to the center of the bore while their large radius characteristic deteriorated much more rapidly. These magnets appear to be of a quality that would yield a chromaticity similar to the present Main Ring.

Figure 4 is a plot of the central dipole field as a function of the distance along the central axis. This data was measured by two methods, first with a hall probe and then with the rotating probe. The data for the rotating probe is the relative dipole field only.

As can be seen from the data in Table IV, a large quadrupole term was observed. Measurements with the 24" stepped harmonic have shown that roughly $20 \%$ of the quadrupole amplitude can be attributed to a magnetized seam weld on the stainless steel warm bore tube. For the moment the source of the quadrupole is not known and investigation is continuing to find the cause and eliminate it.

Tracking Tests

In order for accelerator magnets to be dynamically useful, their fields must reproduce from magnet to magnet and from pulse to pulse. That is to say, at any current and current ramp the ratio of fields of any two magnets is a constant. To measure the tracking of the Dual Dipoles the circuit in Figure 4 was used. Long pickup coils, matched mechanically, were placed in each magnet and connected so that their outputs bucked. Further improvement of the match was accomplished by rotating the coils with respect to their mutual axis for a minimum output while exciting the Dual Dipoles with a low amplitude $60 \mathrm{~Hz}$ signal while the magnets were warm. The actual test was accomplished by integrating the difference signal and displaying this on an $\mathrm{x}-\mathrm{y}$ recorder against the integral of only one of the pickup coils. This gave a plot of differentual flux vs. total flux, $2\left(\Phi_{A}-\Phi_{B}\right) /\left(\Phi_{A}+\Phi_{B}\right)$. Because 
the match between the pickup coils was not perfect the resultant display is a line of positive slope, the linearity of which represents the match of the two magnets. In order to quantitatively measure the tracking difference between the two dual dipoles, each of the output curves was fitted by least squares method to the linear expression:

$$
\Delta \Phi=\mathrm{a}+\mathrm{b} \Phi, \text { where } \Delta \Phi=\Phi_{\mathrm{A}}-\Phi_{\mathrm{B}} \text {. }
$$

The residuals, which show the departure from linearity, then give a quantative measure of dynamic tracking. After folding in the integrator gains and recorder sensitivities, one can set an upper limit on the mismatch of $~ 5$ parts in $10^{4}$. This data, taken for 100,50 and 30 second cycles is summarized in Table V. One could attempt to model and fit higher order terms in $\Phi$ to show the form of the departure from linearity. However, since the magnitudes of the residuals are approximately the same as the effects we are examining, it did not seem worth further investigation. The average residual is then a measure of the error bandwidth and sets an upper limit on tracking error. Summary

The tests reported above mark completion of a necessary stage of development towards a working Energy Doubler dipole. They have shown that two magnets can be constructed with dc and ac excitation characteristics sufficiently alike to be used in an accelerator. Ramp rate sensitivity of the preliminary superconducting wire indicated no cause for concern about the wire to be used in the 4.5 Tesla, 6 meter Doubler dipoles. Further refinements in calculation of wire placement as a result of chromaticity requirements for the Energy Doubler accelerator will have to be undertaken but these magnets, apart from the apparently anomolous quadrupole component, meet the specifications put on them - namely, field homogeneity to $<1$ part in $10^{3}$ at $70 \%$ of coil radius.

Further modeling studies are scheduled only on full scale prototype magnets wound with the final conductor. In addition to repeating field quality and operational performance measurements, future tests will determine energy loss per cycle due to ramping of the magnetic field and other cryogenic losses. An important part of the continuing program will be the testing at 
full field of the cryostat suspensions and the magnet banding system since the present tests have only loaded them to one quarter of their specified value.

Acknowledgements

The authors would like to acknowledge the Technical Services Section who built the magnet and in particular John Stull, George Biallas, Rolf Brocker and each of the magnet facility technicians who worked on this project. We would also like to thank Paul Reardon and Don Edwards for their support, encouragement and many helpful discussions. We owe a special debt to Ernie Ioriatti and Ed Dreier who participated in all the building and testing stages of this project, who put up with our many whims and without whose help we would be six months behind.

References

1. "A Magnetically Operated Cryogenic Valve", M. A. Otavka, B. P. Strauss, R. W. Fast, Advances in Cryogenic Engineering, Vol. 16.

2. Rawson-Lush rotating coil gaussmeter, manufactured by Rawson-Lush Instrument Co., Inc., Acton, Massachusetts.

3. "Spectroscopy of Quadrupole Magnets", J. Cobb and R. Cole, SLAC-PUB133, Sept. 1965.

4. "Determination of the Quality of Multiple Magnets", J. Cobb, A. Robb, and D. Jensen, SLAC-PUB-313, June 1967.

5. "A New Precision Measurement System for Beam Transport Type Magnets", J. Cobb and D. Horelick, SLAC-PUB-750, May 1970.

6. "A Rotating Coil Device for Harmonic Analysis of Magnetic Fields", R. Juhala and G. Michelassi, NAL TM-455, Nov. 6, 1973.

7. "Stationary Coil for Measuring the Harmonics in Pulsed Transport Magnets", G. H. Morgan, Proceedings of the 4th International Conference on Magnet Technology, 1972; USAEC, CONF-720908; also Brookhaven National Laboratory paper BNL 17196.

8. "Stepped Motion, $\cos \mathrm{n} \theta$ Wound Pickup Coils for Harmonic Analysis of Magnetic Fields", B. P. Strauss and D. F. Sutter, National Accelerator Laboratory Technical Memo \# TM-457, January, 1974 (to be published). 
Table I

DUAL DIPOLE MAGNET-PARAMETERS

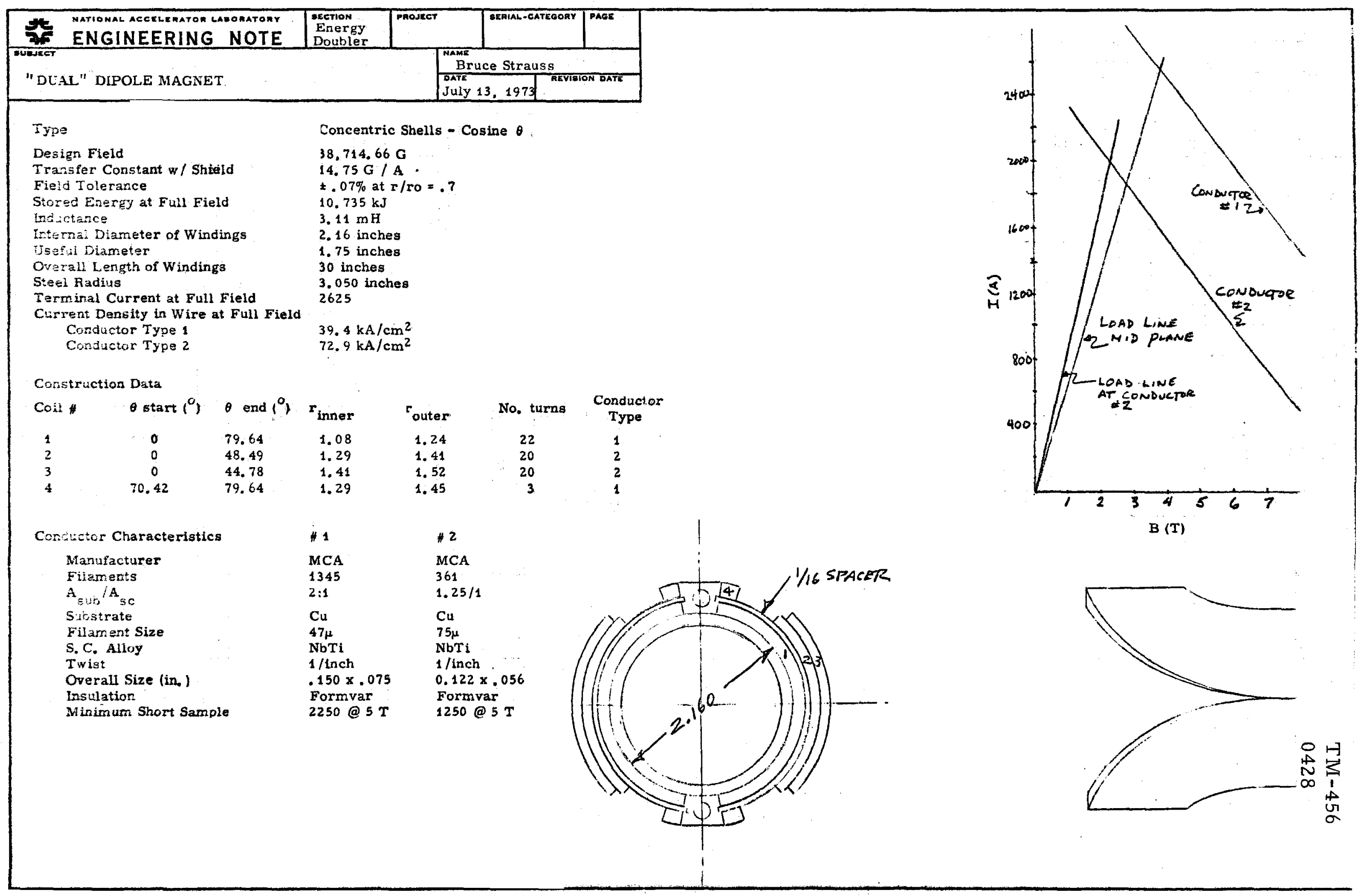


Table II

\section{MAGNET PERFORIMANCE}

Maximum Current

Maximum Field at Center

Transfer Constant with Shield

Right side

Left side

Predicted
$1620 \mathrm{~A}$

$23,959 \mathrm{G}$

14. $785 \mathrm{G} / \mathrm{A}$

14. $809 \mathrm{G} / \mathrm{A}$

14. $75 \mathrm{G} / \mathrm{A}$

$0.16 \%$

$0.20 \%$

Conductor $1\left(.150^{\prime \prime} \mathrm{x} .075^{\prime \prime}\right) \quad 22.32 \mathrm{kA} / \mathrm{cm}^{2}$

Conductor $2\left(.122^{\prime \prime} \mathrm{x} .056^{\prime \prime}\right)^{*} 36.75 \mathrm{kA} / \mathrm{cm}^{2}$

* Maximum central field limited by their "off the shelf" wire

INSTRUMENTAL ERROR OF ROTATING HARMONIC COIL

Relative Amplitude to Dipole

$2 \theta$

$3 \theta$

$5 \theta$

$7 \theta$
$7.33 \times 10^{-4}$

2. $76 \times 10^{-4}$

$2.00 \times 10^{-4}$

$\mathrm{Nil}$ 
Table IV

HARMONIC COEFFICIENTS $\left(a_{n}\right)$

\begin{tabular}{c|ccc|cc|cc} 
& \multicolumn{2}{|c|}{ Right Side Magnet } & \multicolumn{2}{c|}{ Left Side Magnet } & \multicolumn{2}{c}{ Computed } \\
\hline$\theta$ & rotating & $\begin{array}{c}\text { stepped } \\
9^{\prime \prime}\end{array}$ & $\begin{array}{c}\text { stepped } \\
24^{\prime \prime}\end{array}$ & rotating & $\begin{array}{c}\text { stepped } \\
9^{\prime \prime}\end{array}$ & Biallas & Snowden \\
\hline $2 \theta$ & .00238 & .00287 & .00248 & .00517 & .00364 & - & - \\
$3 \theta$ & .00193 & .00086 & .00560 & .00197 & .00960 & -.00082 & .00021 \\
$5 \theta$ & .00487 & .00144 & .00320 & .00609 & .00816 & .00209 & .00197 \\
$7 \theta$ & .0206 & $\mathrm{NM}$ & $\mathrm{NM}$ & .01812 & .01845 & .0201 & .01845
\end{tabular}

Dimension for each $a_{n}$ are in (inches) $-(n-1)$

(NIM $=$ not measured) 
TM -456

0428

Table V

TRACKING TESTS

\begin{tabular}{ccccc}
$\begin{array}{l}\text { Power cycle } \\
\text { time }(\mathrm{sec})\end{array}$ & $\begin{array}{l}\text { Average } \\
\text { residual }\end{array}$ & $\mathrm{b}$ & $\mathrm{a}$ & $\frac{\mathrm{dB}}{\mathrm{dt}}\left(\frac{\mathrm{G}}{\mathrm{sec}}\right)$ \\
\hline 100 & $5 \times 10^{-4}$ & .4079 & .0018 & 444 \\
50 & $5 \times 10^{-4}$ & .4091 & .0165 & 888 \\
$\begin{array}{c}\text { composite of } \\
33,42,50\end{array}$ & $8 \times 10^{-4}$ & .4066 & .0301 & $1347,1110,888$
\end{tabular}

Table V: The equation fitted is $\Delta \Phi=\mathrm{a}+\mathrm{b} \Phi$ where $\Delta \Phi=\Phi_{\mathrm{A}}-\Phi_{\mathrm{B}}$ and $\Phi=\Phi_{A} \simeq \Phi_{B}$. The composite runs were all superimposed on the recorder and could not be individually resolved by this analysis. The slightly larger average residual reflects the scatter of 3 runs - i. e. the line width. 


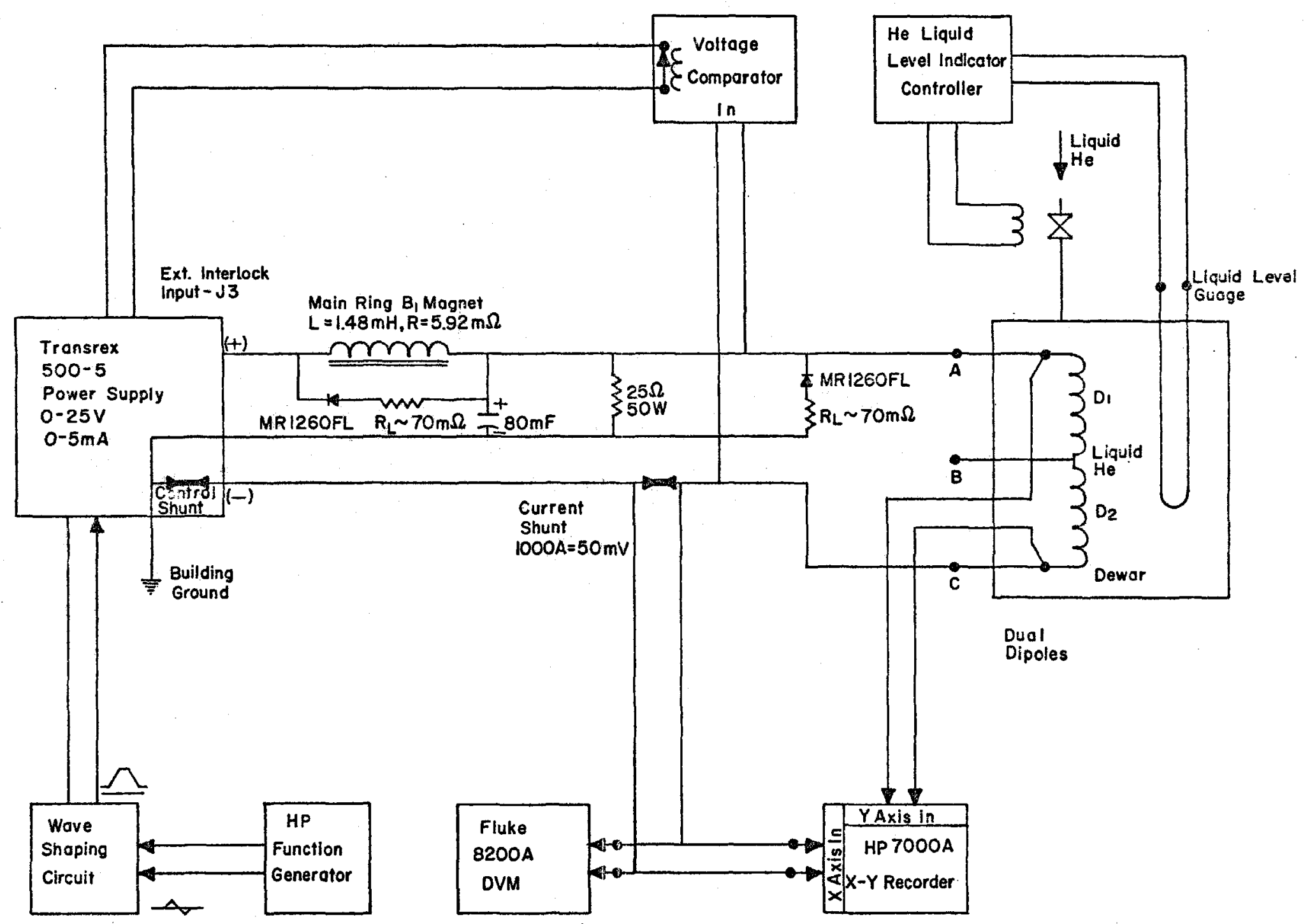

是是

Figure 1 


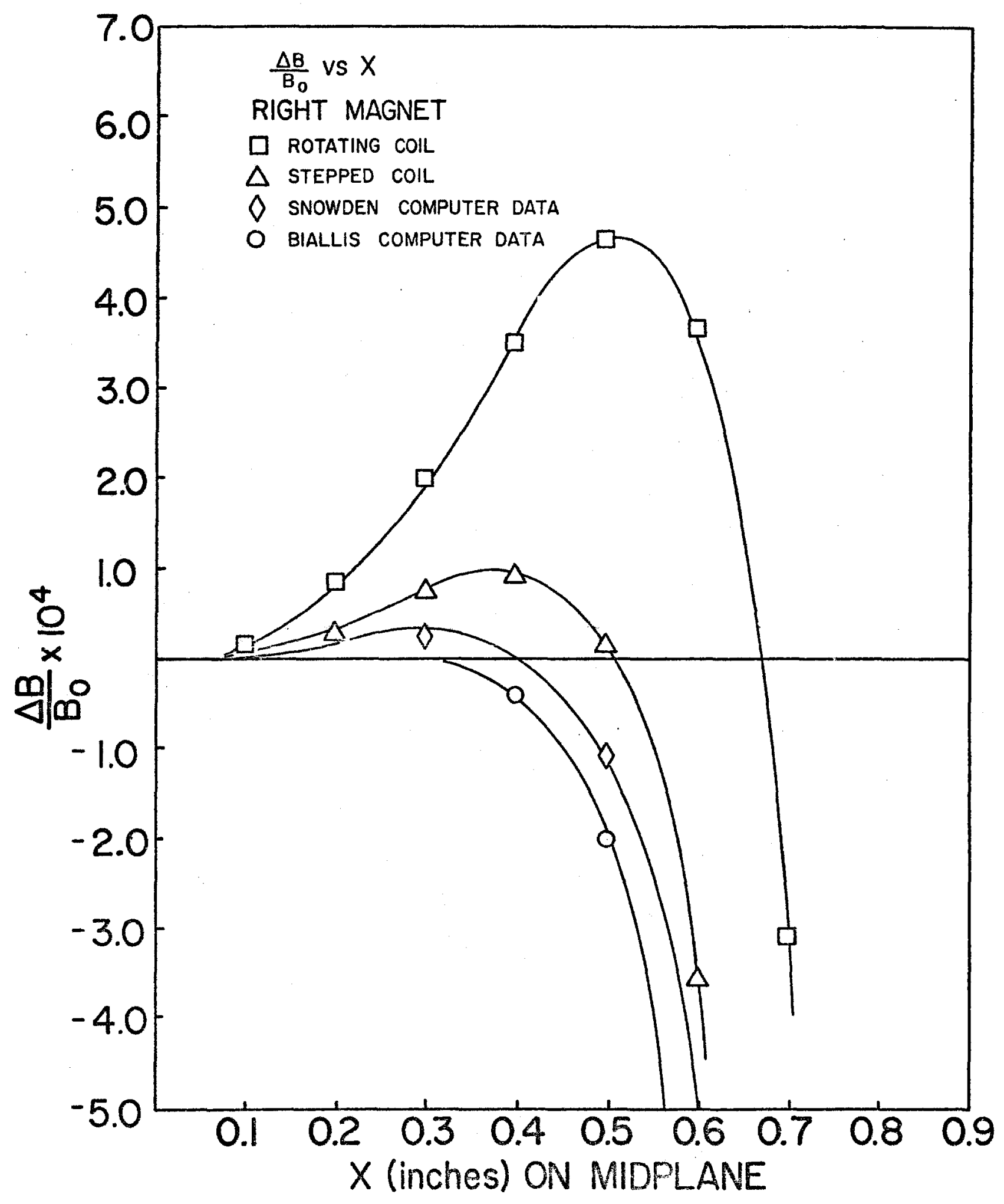

Figure 2 
TM-456

0428

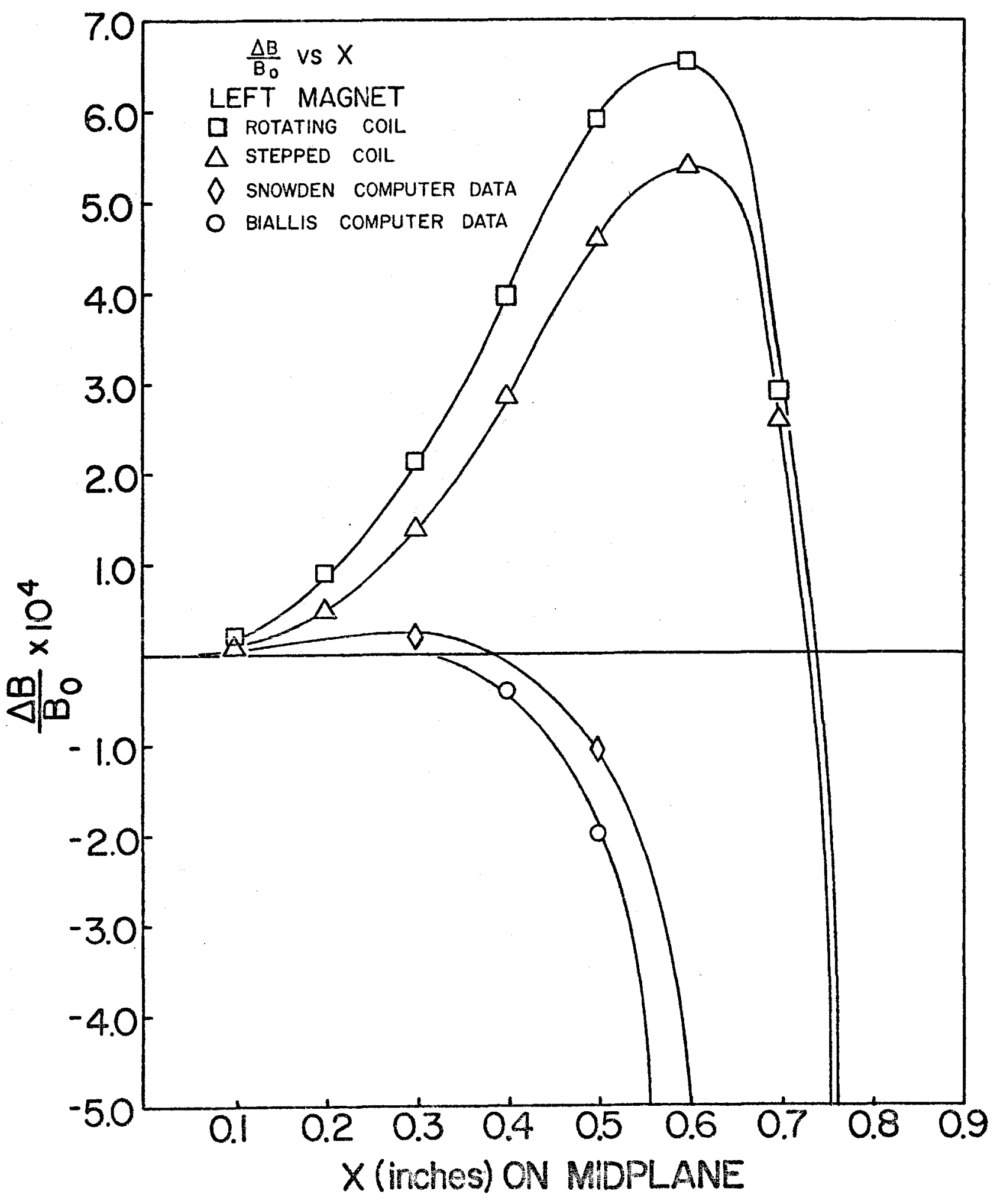

Figure 3 


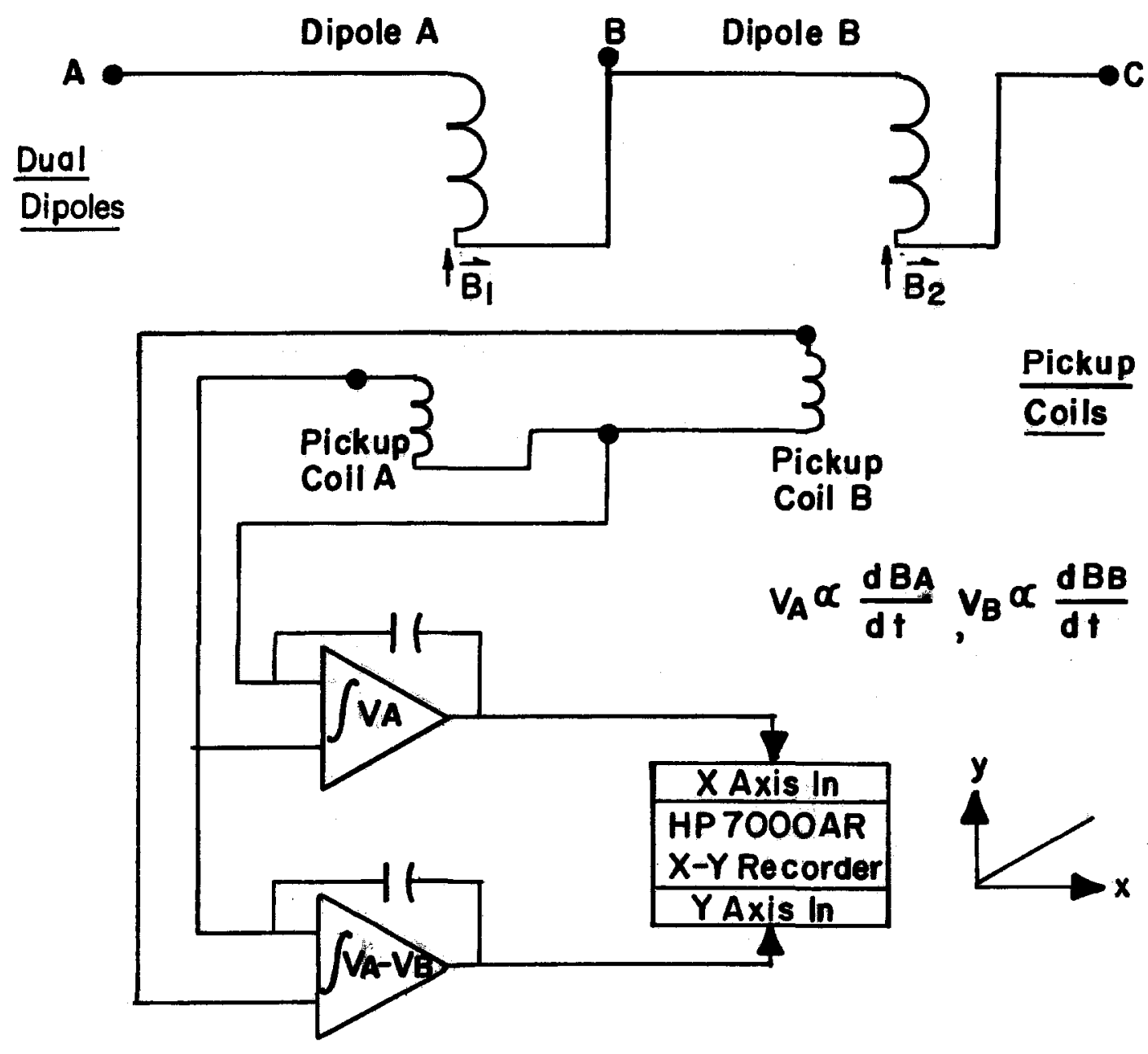

Figure 5

Dual Dipole Tracking Test-Instrumentation 